DATASET Brief

\title{
Identification of proteins in human substantia nigra
}

\author{
Efstathia Kitsou ${ }^{1}$, Sheng Pan ${ }^{1}$, JianPeng Zhang ${ }^{1}$, Min Shi ${ }^{1}$, Aram Zabeti ${ }^{1}$, \\ Dennis W. Dickson ${ }^{2}$, Roger Albin ${ }^{3}$, Marla Gearing ${ }^{4}$, Daniel T. Kashima ${ }^{1}$, Yan Wang ${ }^{1}$, \\ Richard P. Beyer ${ }^{5}$, Yong Zhou ${ }^{6}$, Catherine Pan ${ }^{1}$, W. Michael Caudle ${ }^{1}$ and Jing Zhang ${ }^{1}$ \\ ${ }^{1}$ Department of Pathology, University of Washington School of Medicine Seattle, Seattle WA, USA \\ ${ }^{2}$ Department of Pathology, Mayo Clinic, FL, USA \\ ${ }^{3}$ Department of Neurology, University of Michigan, and Geriatrics Research, \\ Education, and Clinical Center, Ann Arbor VAHS, MI, USA \\ ${ }^{4}$ Department of Neurology, Emory University, GA, USA \\ ${ }^{5}$ Environmental and Occupational Health Science, University of Washington, Seattle, WA, USA \\ ${ }^{6}$ Institute for Systems Biology, Seattle, WA, USA
}

Characterization of the human brain proteome is a critical area of research. While examination of the human cortex has provided some insight, very little is known about the proteome of the human midbrain, which demonstrates substantial loss of dopaminergic neurons in the substantia nigra pars compacta (SNpc) in Parkinson's disease (PD). Therefore, characterization of this region is essential to a better understanding of the pathogenesis of PD. This dataset paper reports two separate studies, where human SNpc was collected from PD and control subjects and 1263 proteins were identified using MALDI-TOF/TOF as well as linear ion trap MS platforms. With gene ontology analysis, the proteins were categorized according to their biological processes, as well as cellular components. These data were also compared with previous proteomic characterization of the human frontal and temporal cortex, and cerebrospinal fluid to establish shared proteins of relevance. The present dataset is the most extensive survey of the human SNpc proteome, to date. Further characterization of the SNpc proteome will significantly facilitate our understanding of the function and expression of proteins involved in PD, as well as provide potential proteins that may be utilized as biomarkers.

\section{Keywords:}

Biomarkers / Cerebrospinal fluid / MS / Parkinson's disease / Substantia nigra pars compacta
Received: January 22, 2008

Accepted: January 23, 2008

\footnotetext{
Correspondence: Professor Jing Zhang, Division of Neuropathology, Harborview Medical Center, University of Washington School of Medicine, BOX 359635, 325 9th Avenue, Seattle, WA 98104-2499, USA

E-mail: zhangj@u.washington.edu

Fax: +1-206-341-5249
}

Abbreviations: CSF, cerebrospinal fluid; EBI, European Bioinformatics Institute; ICAT, isotope coded affinity tag; IPI, International Protein Index; LTQ, linear ion trap MS; PD, Parkinson's disease; SCX, strong cation exchange chromatography; SNpc, substantia nigra pars compacta; VTA, ventral tegmental area
Since the completion of the human genome project, it has become clear that characterization of the human proteome is indispensable to our understanding of the biological function and processes of proteins, both in a normal as well as a pathological state. Recently, we, as well as others, have begun to identify proteins that are unique to certain brain regions in order to characterize proteins that may qualitatively change under pathological conditions [1-3]. For example, proteomic analysis of human frontal cortex that is typically involved in cognitive function and geriatric dementia (using a platform of biochemical fractionation, multi- 
dimensional LC separation, and a MALDI-TOF/TOF mass spectrometer) identified over 800 proteins, of which several were found to be associated with neurodegenerative disease [1]. Furthermore, examination of the human temporal lobe, a region often involved in epilepsy (using 2-DE separation with MALDI-TOF/TOF [4] as well as a shotgun proteomics approach that utilized strong cation exchange (SCX), RP-LC and 3-D ion trap mass spectrometry [5]) has identified several hundred proteins, including many linked to epilepsy.

The focus of this dataset paper is the midbrain whose degeneration is linked to multiple movement disorders, including Parkinson's disease (PD), multiple system atrophy, and progressive supranuclear palsy. The human midbrain is composed of several different regions, including the superior and inferior colliculi, red nucleus, substantia nigra pars reticulata, ventral tegmental area (VTA), and substantina nigra pars compacta (SNpc). It is the degeneration of dopaminecontaining neurons in the $\mathrm{SNpc}$ that underlies the motor deficits that characterize most movement disorders [6]. In an earlier study of human midbrain we utilized isotope coded affinity tag (ICAT) labeling of mitochondrial-enriched proteins from the SNpc of human PD and control brains, followed by analysis with linear ion trap MS (LTQ) [7]. Comparison of the protein profiles in the SNpc of PD patients with those of age-matched controls identified 842 proteins, of which 119 proteins displayed changes in relative abundance in the diseased tissue $v$ s. controls [7]. These data are a much larger representation of the $\mathrm{SNpc}$ proteome than has been previously demonstrated in other studies. For example, examination of SNpc of human PD and control samples through 2-DE identified 44 proteins, of which nine showed changes in expression between control and disease brains [8].

As a part of an ongoing effort to further characterize and understand the human midbrain proteome as well as identify alterations in protein expression levels in a normal and pathological state, we have recently compared the proteome of the SNpc with another midbrain region, the VTA that is much less sensitive to neurodegeneration in PD. While proteins displaying quantitative changes are being evaluated extensively (and will be published separately), the results on protein identification in the midbrain is reported here, along with proteins identified previously by us [7] after standardization of the data processing. We hope that making these data available to other investigators in the field can facilitate the process of characterization of the human midbrain, comprehensively. To make our dataset even more user-friendly, we will make it publicly accessible on our website as well as the PRIDE database managed by the European Bioinformatics Institute (EBI). Finally, we hope that this dataset will contribute to the current initiatives that are supported by the National Institutes of Health (Brain Molecular Anatomy project: http://trans.nih.gov/bmap/) as well as the Human Proteome Organization (www.hupo. org).

Technical details are provided for three major aspects of the investigation: (i) Sample preparation: Samples from SNpc and VTA of human midbrain were obtained at autopsy according to protocols approved by the University of Washington School of Medicine, Mayo Clinic in Florida, University of Michigan, and Emory University. The average age of the subjects was 76 years old and postmortem interval was less than $12 \mathrm{~h}$. All control subjects (four males and one female; average age: 82 years) were individuals who were not diagnosed with a neurological disease and whose neuropathological examination revealed age-related changes only. All PD patients (five males of an average age of 70 years) had been clinically diagnosed with an extrapyramidal movement disorder or dementia, and their final diagnoses were established by neuropathological examination according to established criteria $[9,10]$. Four groups of samples were analyzed: Control VTA $(n=5)$, Control SNpc $(n=5)$, PD VTA $(n=5)$, and PD $\operatorname{SNpc}(n=5)$.

Frozen samples were rapidly thawed in ice-cold homogenization buffer (0.32 M sucrose, $20 \mathrm{mM}$ HEPES, pH 7.5, $1 \mathrm{mM}$ PMSF, phosphatase inhibitors $\left(0.2 \mathrm{mM} \mathrm{Na}_{3} \mathrm{VO}_{4}\right.$ and $1 \mathrm{mM} \mathrm{NaF}$ ) and protease inhibitor cocktail from Sigma, St. Louis, MO), and then disrupted with a glass-Teflon homogenizer (Wheaton, Millville, NJ) by ten gentle up and down strokes. The protein concentrations of the homogenates were determined using a Pierce BCA Protein Assay kit (Rockford, IL). Equal amount of proteins from five individual samples in each group were combined together. Cytosolic, mitochondrial and nuclear fractions of SNpc were prepared as previously described [1]. Fractionation of tissue was not designed to achieve pure sub-cellular organelles, as this is not practically possible for frozen human brain. Rather, this procedure was instituted for deeper probing of the human proteome.

(ii) Protein identification and quantification: For proteins in each of the enriched fractions in the four groups, i.e. control VTA, control SNpc, PD VTA, and PD SNpc, this was achieved using the iTRAQ labeling technique that is routinely utilized in our lab [11]. In this study, for each enriched fraction, $100 \mu \mathrm{g}$ of proteins from each group were tryptically digested in parallel and then labeled with iTRAQ reagents (Applied Biosystem, Foster City, CA) according to the manufacture's protocol. The labeled digests from the four groups were combined together and further separated into nine fractions using an offline SCX PolySulfoethyl ATM column (200 mm $\times 2.1 \mathrm{~mm}$ id, 5 micron, $300 \AA$, Poly LC, Columbia, $\mathrm{MD})$. Of note again, results related to quantitative changes among different regions and/or disease vs. controls will be reported separately.

Each SCX fraction was collected and further separated by RP nanocapillary LC (LC Packing/Dionex, Sunnyvale, CA). The mass spectrometric analysis was carried out using a MALDI-TOF/TOF instrument (4700 Proteomics Analyzer, Applied Biosystems) with reflector positive ion mode and each fraction was analyzed in triplicate. Spectral results were searched against the International Protein Index (IPI) human protein database (version 3.18) from the EBI using the 
GPS software (version 3.6, Applied Biosystems) running MASCOT search algorithm (version 2.0, Matrix Science, Boston, MA) for peptide and protein identification. All proteins identified by a decoy database (less than $2 \%$ of total proteins) were eliminated from the list of proteins reported.

It should be noted that the data related to ICAT and LTQ analysis of SNpc has been reported previously as an appendix associated with a biology paper [7]. A few key elements are repeated here for the readers' reference and standardization of the dataset. Briefly, proteins were identified in the SNpc of control and PD patients using the ICAT labeling technique, initially described by Gygi et al., [12] and routinely used in our lab [1, 3, 13]. For the LTQ platform, peptides were separated and analyzed on an LTQ work station (Thermo Electron, San Jose, CA) using automated, on-line 2-D LC separation followed by nanoelectrospray LC/MS [14]. Proteins from the mixture were later identified automatically using the computer program SEQUEST, which searched the MS/MS spectra against the human IPI database (version 2.33 in initial publication, but reprocessed with version 3.18) database $[3,13]$. Potential peptides and proteins were further analyzed with two newly developed computer software programs, PeptideProphet and ProteinProphet based on statistical models [15]. The list of proteins filtered by ProteinProphet typically has very little overlap (less than 1\%) with that searched with a decoy database [16].

(iii) Bioinformatics: The Gene Ontology (GO) analysis was carried out at the Bioinformatics and Biostatistics Facility Core of the University of Washington. All proteins identified were analyzed by GO for protein categorization [17]. Briefly, queries using the IPI values were submitted to the EMBL-EBI IPI database using the Bioconductor [18] software package. Bioconductor commands were used to construct and send dbfetch queries to the IPI database via a URL, for example, http://www.ebi.ac.uk/cgi-bin/dbfetch?db=IPI\$id= IPI00298853. These IPI query results were further processed in Bioconductor to construct a master table containing IPIs, Entrez Gene IDs, Gene Symbols, and Gene Descriptions. The Entrez Gene IDs were used in conjunction with the Bioconductor biomart package [19] to query the Ensembl BioMart database for the GO IDs as well as GO categories of the three GOs (Biological Process, Molecular Function, Cellular Component). Because any Entrez Gene ID can occur in more than one GO category, the calculation of the numbers of proteins in selected GO categories, whether cellular component or biological process, was done on a priority basis, meaning that a gene/protein was removed from the master table after it was classified so that multiple counting of the gene/proteins was avoided.

As mentioned previously, the proteins identified in the $\mathrm{SNpc}$ in our investigations are the most extensive thus far. A few key technical and biological issues unique to this dataset are discussed further as the following:

(i) Protein identification and GO analysis: All current MS-based proteomics is biased towards identification of proteins of high abundance [20] thus, it is critical to fractionate tissue homogenates to achieve a deeper analysis of the cellular proteome. Moreover, through this approach, it is more likely to identify the proteins whose total cellular levels remain the same but relative levels in specific cellular compartments change during processes. For example, in our previous ICAT analysis of PD SNpc versus control SNpc, mortalin was found to be decreased in mitochondrial but not cytosolic fractions, a fact further validated by alternative means [7]. Our current study led to the identification of 951 proteins; of those, 399 non-redundant proteins were identified with two or more peptides. The number of non-redundant proteins identified with two or more peptides was 313, 114, and 170 for cytosolic, mitochondrial, and nuclear fractions, respectively (a total of 399 after removing redundant proteins). A second set of proteins was identified using a multidimensional separation strategy in combination with
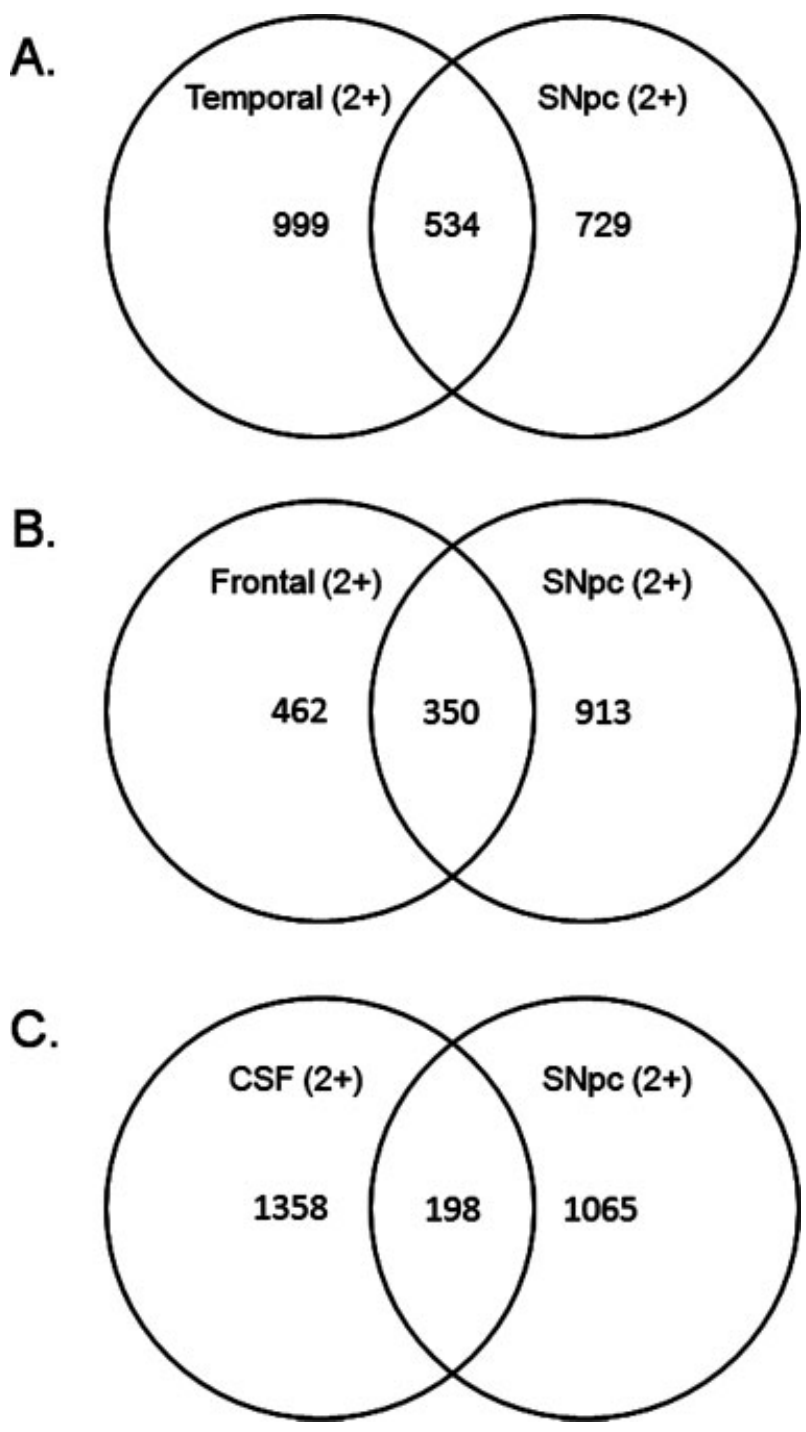

Figure 1. Overlap of the SNpc proteome with the Temporal Lobe (A), Frontal Cortex (B), and CSF (C). Only proteins identified by two or more peptides were included in these comparisons. 
an ESI based ion trap mass spectrometer [7, 21]. Using this approach, 1049 proteins were identified by at least two peptides. It should be noted that previous analysis of this same dataset yielded 842 proteins identified by two or more peptides. This discrepancy in the number of proteins identified can be attributed to the rapid progression of the proteomics field and the updating of the protein database that was originally used. Indeed, our previous investigation has indicated that a significant number of proteins are altered when an identical MS data was searched against two different versions of the IPI database [11].

When MALDI-TOF/TOF protein identification was combined with the LTQ study [7] a total of 1263 non-redundant proteins were identified in the $\mathrm{SNpc}$, which represents the most extensive characterization of the human midbrain to date. Notably, 14 proteins identified previously by a single peptide were seen in the current study with more than two peptides. Conversely, 87 proteins identified in the current study by a single peptide were revealed in the previous investigation by more than two peptides. Nonetheless, to comply with the general rule of proteomic data depository, only proteins identified by more than two peptides in at least one study are reported here. A complete list of the proteins can be found in Supporting Information Table 1.

$\mathrm{SNpc}$ proteins identified by more than two peptides in our study were classified in accordance with their distribution into cellular components and biological processes based on GO analysis and annotations (Supporting Information Fig. 1). Categorization of proteins provides a starting point, from which a more in-depth investigation of individual proteins that show potential involvement in PD can be undertaken. The major cellular components of the proteins identified fall into several categories, including mitochondria, membrane and endoplasmic reticulum/lysosome, and nuclear (Supporting Information Fig. 1A). The main biological functions of the proteins identified in the SNpc include oxidative stress processes, regulation of cytoskeletal organization, as well as participation in cell-cell communication and signaling (Supporting Information Fig. 1B). A significant portion of proteins have various other functions that collectively fall into a category denoted as "others". With the advance of bioinformatics and proteomics technology, it is hoped that a complete characterization of the midbrain proteome will be achieved in the years to come.

(ii) Comparison of the SNpc proteome with other human brain regions and CSF as well as mouse midbrain: Of the 1263 proteins identified in midbrain, 534 have been reported previously in the temporal cortex [24] and 350 in the frontal cortex [1]. The overlap of the proteins identified in each of the proteomes is demonstrated in Fig. 1A and B, respectively. We have also compared our current SNpc dataset with a previous proteomic study that utilized 2-DE and MALDI-TOF-MS to identify 44 proteins in human SNpc [8]. Of these 44 proteins, over $75 \%$ of them were also identified in our study, providing evidence for the validity of our results. In addition, comparison of proteins recently identi- fied by our lab in a proteomic study of mouse midbrain determined overlap between numerous proteins found in both human SNpc and mouse midbrain [3]. Examples of some of these proteins include, HSPs, 14-3-3, thioredoxin, glutathione S-transferase, and ubiquitin carboxy-terminal hydrolase L1 (UCHL-1). These data provide further evidence for the utility of using mice as a reliable model of PD. On the other hand, a significant portion of proteins (over 99\%) identified in human SNpc were not identified in a mouse genomic comparison between the SNpc and VTA [23] emphasizing again that it is imperative to investigate the human CNS proteome (ideally human) directly.

Finally, we compared the proteins identified in the midbrain with those in human cerebrospinal fluid (CSF). CSF is easily accessible and the proteins present in it could be used as biomarkers for neurodegenerative diseases. Furthermore, CSF is a viable source for biomarker discovery because of its proximity to the site of pathology and because it reflects the metabolic state of the brain under varying conditions that effect protein concentrations and patterns in CSF considerably. By comparing the previously identified CSF proteome (a total of 2556 proteins) [2, 11] with that of the SNpc identified in our present study, 198 proteins were found to be shared by the SNpc and CSF proteomes (Fig. 1C). Many of these shared proteins, e.g. calmodulin, $\gamma$-enolase, and myelin basic protein are known to be involved in a variety of CNS functions. While these data represent the most current evaluation of the human SNpc available, we realize that the dynamic and continually progressing nature of proteomic technology may further validate or abrogate these findings.

(iii) Discussion of the proteins identified in the dataset: Among the proteins identified in the SNpc tissue, many of them are functionally or structurally involved in neurodegeneration. Further characterization of these proteins will facilitate a more complete understanding of the pathogenesis of various movement disorders. Proteins that are well known to be critical to movement disorders are listed in Supporting Information Table 2. A significant number of these proteins are involved in the process of mitochondrial function, oxidative stress, protein degradation, and neuroinflammation, processes that have been clearly indicated to be important to neurodegenerative diseases, including PD (e.g. DJ-1, glutathione S-transferase omega1, manganese superoxide dismutase, and UCHL-1). These proteins have been discussed extensively in the literature. Additionally, as one of the most important advantages of nonbiased proteomics centers on discovering novel proteins that are yet to be determined to be critical to a particular disease or disease state, we have decided to focus the remainder of the discussion on a subset of proteins that have not been related to PD pathogenesis thus far, although investigations in other fields appear to suggest their roles in neural functions.

Calbindin: Calbindin D28K protein is a calcium binding protein [24] involved in the regulation of intracellular calcium [25]. Calbindin is expressed in dopamine neurons of the dorsal tier of the $\mathrm{SNpc}$ that project to the limbic region of 
the telencephalon and cerebral cortex [26]. In contrast, dopamine neurons from the ventral tiers of the $\mathrm{SNpc}$ do not contain calbindin and project to the dorsolateral motor putamen [27]. Interestingly, dopamine neurons located in the VTA also express calbindin [28] yet seem to be less vulnerable to PD than dopamine neurons in the SNpc [29]. Furthermore, Calbindin does not appear to demonstrate a neuroprotective role [30] which suggests that other cellular characteristics are involved in mediating the differential vulnerability of dopamine neurons in the VTA and SNpc.

Calmodulin: Calmodulin is a calcium binding protein that binds and modulates several different cellular processes, including inflammation, metabolism, and apoptosis. Our study identified six peptides associated with calmodulin. Of these, two peptides $(91-106,127-148)$ cover the calciumbinding regions. A recent study showed that calmodulin binds to $\alpha$-synuclein in a calcium dependent-manner and accelerates $\alpha$-synuclein fibrillation [31] which has been demonstrated to be a major factor in the pathogenesis of PD. In addition, oxidative stress and mitochondrial dysfunction have been suggested to be involved in the pathogenesis of neurodegenerative diseases, and calmodulin has been linked to both these processes [32]. Finally, a comparative proteomic analysis of striatum from rats exposed to 6-hydroxy-dopamine identified calmodulin as a protein that underwent a change in expression in rats treated with 6-hydroxy-dopamine, compared with control [33].

Cofilin: This protein belongs to the protein family of ADF/cofilins [34] and is an important regulator of actin, a key component of microtubules [35]. Disruption of microtubules and subsequent axonal transport could have detrimental effects on numerous cellular processes integral to the well-being of the cell. Cofilin has been implicated initially in Alzheimer's disease [36] and it is suggested that neurodegenerative stimuli can activate $\mathrm{ADF} /$ cofilin by dephosphorylation, causing the formation of bars which can impair axonal transport along the microtubules, inhibiting neurite function [37]. There is also data showing that 1methyl 4-phenyl 1,2,3,6-tetrahydropyridine (MPTP) treated mice demonstrate changes in the expression of the cytoskeletal genes, including cofilin [38]. Furthermore, parkin, a protein that has been implicated in inherited forms of PD, may interact with LIM kinase-1 and suppress the phosphorylation of cofilin [39].

14-3-3: 14-3-3 proteins, a family of chaperones that participate in many signaling pathways, are abundant in the brain. In this study, six different isoforms of 14-3-3 protein were identified in the SNpc, including beta/alpha, epsilon, eta, gamma, theta, and zeta/delta. There is evidence suggesting that 14-3-3 proteins may play a central role in PD pathogenesis [40] as it has been shown by immunochemical studies to colocalize with Lewy bodies [41]. In addition, it has been shown that one family member, 14-3-3 eta specifically interacts with parkin and inhibits its ubiquitin ligase activity, which could impede proteasomal degradation of damaged or misfolded proteins in the cell [42].
Stathmin: Stathmin is a cytosolic phosphoprotein, abundantly expressed in human brain, playing a role in adult neurogenesis [43]. Stathmin regulates microtubule dynamics [44] and by way of this function it has been suggested by in vitro studies that when overexpressed it could lead to Golgi fragmentation - a process that links stathmin to amyotrophic lateral sclerosis [45]. In addition, stathmin has been associated with Alzheimer's disease and Down's syndrome [46] therefore it could have a role in other neurodegenerative diseases such as PD for which Golgi fragmentation could be a contributing factor in the pathogenesis of neuronal degeneration [47]. Interestingly, a recent study demonstrated Golgi fragmentation in the SNpc of PD patients exhibiting $\alpha$ synuclein positive inclusions [48].

$\gamma$-Synuclein: One of the three members of the human synuclein family, including $\alpha, \beta$-synuclein, $\gamma$-synuclein shows $60 \%$ similarity with $\alpha$-synuclein at the amino acid sequence level [49] and is expressed throughout the brain, particularly in the substantia nigra [50]. In contrast to $\alpha$ synuclein, which has been extensively demonstrated to be involved in the pathogenesis of PD, the role of $\gamma$-synuclein in PD is still disputable. Although studies have demonstrated a lack of specific polymorphisms in the $\gamma$-synuclein gene, several studies have shown potential aggregations of $\gamma$-synuclein in neuronal structures [51]. More recently, it has been shown that $\gamma$-synuclein is able to inhibit $\alpha$-synuclein fibrillation, a potentially imperative step in $\alpha$-synuclein-mediated PD pathology [52].

In summary, our work provides an inventory of proteins that are linked to neurodegenerative processes. The expressed proteins need to be validated and more thoroughly characterized to elucidate their function. It is important to recognize that the $\mathrm{SNpc}$ is not a homogeneous structure. In primates, the dopaminergic $\mathrm{SNpc}$ neurons are interdigitated with GABAergic substantia nigra pars reticulata neurons. Within the SNpc, some groups of dopaminergic neurons are more affected than others by whatever processes cause neurodegeneration in PD [53]. In the future, more fine grained analyses will be needed to assess protein changes in subpopulations of SNpc neurons. Given the complexity of proteomic research and the limited access to brain tissue from patients and healthy individuals this preliminary protein database of the human SNpc could be the first step towards early diagnosis and development of therapeutic targets. Additionally, proteins present in both tissue and CSF may provide tissue specific markers for PD diagnosis and progression.

The research is supported by following grants funded to several investigators in multiple medical centers (R01AG025327, R01ES012703, AG08671 and AG025688) as well as the American Parkinson's Disease Association and a Shaw Endowment to Dr. Jing Zhang.

The authors have declared no conflict of interest. 


\section{References}

[1] Pan, S., Shi, M., Jin, J., Albin, R. L. et al., Proteomics identification of proteins in human cortex using multi-dimensional separations and MALDI tandem mass spectrometer. Mol. Cell. Proteomics 2007, 6, 1818-1823.

[2] Pan, S., Zhu, D., Quinn, J. F., Peskind, E. R. et al., A combined dataset of human cerebrospinal fluid proteins identified by multi-dimensional chromatography and tandem mass spectrometry. Proteomics 2007, 7, 469-473.

[3] Jin, J., Meredith, G. E., Chen, L., Zhou, Y. et al., Quantitative proteomic analysis of mitochondrial proteins: relevance to Lewy body formation and Parkinson's disease. Brain Res. Mol. Brain Res. 2005, 134, 119-138.

[4] He, S., Wang, Q., He, J., Pu, H. et al., Proteomic analysis and comparison of the biopsy and autopsy specimen of human brain temporal lobe. Proteomics 2006, 6, 4987-4996.

[5] Dumont, D., Noben, J. P., Verhaert, P., Stinissen, P., Robben, J., Gel-free analysis of the human brain proteome: Application of liquid chromatography and mass spectrometry on biopsy and autopsy samples. Proteomics 2006, 6, 49674977.

[6] Ehringer, H., Hornykiewicz, O., Distribution of noradrenaline and dopamine (3-hydroxytyramine) in the human brain and their behavior in diseases of the extrapyramidal system. Klin. Wochenschr. 1960, 38, 1236-1239.

[7] Jin, J., Hulette, C., Wang, Y., Zhang, T. et al., Proteomic identification of a stress protein, mortalin/mthsp70/GRP75: Relevance to Parkinson disease. Mol. Cell. Proteomics 2006, 5, 1193-1204.

[8] Basso, M., Giraudo, S., Corpillo, D., Bergamasco, B. et al., Proteome analysis of human substantia nigra in Parkinson's disease. Proteomics 2004, 4, 3943-3952.

[9] Mirra, S. S., The CERAD neuropathology protocol and consensus recommendations for the postmortem diagnosis of Alzheimer's disease: A commentary. Neurobiol. Aging 1997, 18 , S91-S94.

[10] McKeith, I. G., Galasko, D., Kosaka, K., Perry, E. K. et al., Consensus guidelines for the clinical and pathologic diagnosis of dementia with Lewy bodies (DLB): report of the consortium on DLB international workshop. Neurology $1996,47,1113-1124$.

[11] Abdi, F., Quinn, J. F., Jankovic, J., McIntosh, M. et al., Detection of biomarkers with a multiplex quantitative proteomic platform in cerebrospinal fluid of patients with neurodegenerative disorders. J. Alzheimers Dis. 2006, 9, 293-348.

[12] Gygi, S. P., Rist, B., Gerber, S. A., Turecek, F. et al., Quantitative analysis of complex protein mixtures using isotopecoded affinity tags. Nat. Biotechnol. 1999, 17, 994-999.

[13] Zhang, J., Goodlett, D. R., Peskind, E. R., Quinn, J. F. et al., Quantitative proteomic analysis of age-related changes in human cerebrospinal fluid. Neurobiol. Aging 2005, 26, 207227.

[14] Chelius, D., Zhang, T., Wang, G., Shen, R. F., Global protein identification and quantification technology using twodimensional liquid chromatography nanospray mass spectrometry. Anal. Chem. 2003, 75, 6658-6665.

[15] Keller, A., Nesvizhskii, A. I., Kolker, E., Aebersold, R., Empirical statistical model to estimate the accuracy of peptide identifications made by MS/MS and database search. Anal. Chem. 2002, 74, 5383-5392.
[16] Jin, J., Li, G. J., Davis, J., Zhu, D. et al., Identification of novel proteins associated with both alpha-synuclein and DJ-1. Mol. Cell. Proteomics 2007, 6, 845-859.

[17] Camon, E., Magrane, M., Barrell, D., Lee, V. et al., The gene ontology annotation (GOA) database: Sharing knowledge in Uniprot with Gene Ontology. Nucleic Acids Res. 2004, 32, D262-D266.

[18] Gentleman, R. C., Carey, V. J., Bates, D. M., Bolstad, B. et al., Bioconductor: open software development for computational biology and bioinformatics. Genome Biol. 2004, 5, R80.

[19] Durinck, S., Moreau, Y., Kasprzyk, A., Davis, S. et al., BioMart and bioconductor: A powerful link between biological databases and microarray data analysis. Bioinformatics 2005, 21, 3439-3440.

[20] Washburn, M. P., Wolters, D., Yates, J. R., 3rd Large-scale analysis of the yeast proteome by multidimensional protein identification technology. Nat. Biotechnol. 2001, 19, 242247

[21] Xu, J., Chen, J., Peskind, E. R., Jin, J. et al., Characterization of proteome of human cerebrospinal fluid. Int. Rev. Neurobiol. 2006, 73, 29-98.

[22] Park, Y. M., Kim, J. Y., Kwon, K. H., Lee, S. K. et al., Profiling human brain proteome by multi-dimensional separations coupled with MS. Proteomics 2006, 6, 4978-4986.

[23] Pavlidis, P., Noble, W. S., Analysis of strain and regional variation in gene expression in mouse brain. Genome Biol. 2001, 2, RESEARCH0042.

[24] Persechini, A., Moncrief, N. D., Kretsinger, R. H., The EFhand family of calcium-modulated proteins. Trends $\mathrm{Neu}$ rosci. 1989, 12, 462-467.

[25] Baimbridge, K. G., Celio, M. R., Rogers, J. H., Calcium-binding proteins in the nervous system. Trends Neurosci. 1992, 15, 303-308.

[26] Haber, S. N., Ryoo, H., Cox, C., Lu, W., Subsets of midbrain dopaminergic neurons in monkeys are distinguished by different levels of mRNA for the dopamine transporter: comparison with the mRNA for the D2 receptor, tyrosine hydroxylase and calbindin immunoreactivity. J. Comp. Neurol. 1995, 362, 400-410.

[27] Gerfen, C. R., Baimbridge, K. G., Miller, J. J., The neostriatal mosaic: compartmental distribution of calcium-binding protein and parvalbumin in the basal ganglia of the rat and monkey. Proc. Natl. Acad. Sci. USA 1985, 82, 8780-8784.

[28] Thompson, L., Barraud, P., Andersson, E., Kirik, D., Bjorklund, A., Identification of dopaminergic neurons of nigral and ventral tegmental area subtypes in grafts of fetal ventral mesencephalon based on cell morphology, protein expression, and efferent projections. J. Neurosci. 2005, 25, 64676477.

[29] Damier, P., Hirsch, E. C., Agid, Y., Graybiel, A. M., The substantia nigra of the human brain. I. Nigrosomes and the nigral matrix, a compartmental organization based on calbindin $\mathrm{D}(28 \mathrm{~K})$ immunohistochemistry. Brain 1999, 122, 1421-136.

[30] Airaksinen, M. S., Thoenen, H., Meyer, M., Vulnerability of midbrain dopaminergic neurons in calbindin-D28k-deficient mice: lack of evidence for a neuroprotective role of endogenous calbindin in MPTP-treated and weaver mice. Eur. J. Neurosci. 1997, 9, 120-127. 
[31] Martinez, J., Moeller, I., Erdjument-Bromage, H., Tempst, P., Lauring, B., Parkinson's disease-associated alpha-synuclein is a calmodulin substrate. J. Biol. Chem. 2003, 278, 1737917387.

[32] Lee, C. S., Han, E. S., Han, Y. S., Bang, H., Differential effect of calmodulin antagonists on MG132-induced mitochondrial dysfunction and cell death in PC12 cells. Brain Res. Bull. 2005, 67, 225-234.

[33] Pierson, J., Norris, J. L., Aerni, H. R., Svenningsson, P. et al., Molecular profiling of experimental Parkinson's disease: direct analysis of peptides and proteins on brain tissue sections by MALDI mass spectrometry. J. Proteome Res. 2004, 3, 289-295.

[34] Bamburg, J. R., Proteins of the ADF/cofilin family: essential regulators of actin dynamics. Annu. Rev. Cell Dev. Biol. 1999, $15,185-230$.

[35] Kiuchi, T., Ohashi, K., Kurita, S., Mizuno, K., Cofilin promotes stimulus-induced lamellipodium formation by generating an abundant supply of actin monomers. J. Cell Biol. 2007, 177, 465-476.

[36] Heredia, L., Helguera, P., de Olmos, S., Kedikian, G. et al., Phosphorylation of actin-depolymerizing factor/cofilin by LIM-kinase mediates amyloid beta-induced degeneration: a potential mechanism of neuronal dystrophy in Alzheimer's disease. J. Neurosci. 2006, 26, 6533-6542.

[37] Minamide, L. S., Striegl, A. M., Boyle, J. A., Meberg, P. J., Bamburg, J. R., Neurodegenerative stimuli induce persistent ADF/cofilin-actin rods that disrupt distal neurite function. Nat. Cell Biol. 2000, 2, 628-636.

[38] Cuadrado-Tejedor, M., Sesma, M. T., Gimenez-Amaya, J. M., Ortiz, L., Changes in cytoskeletal gene expression linked to MPTP-treatment in Mice. Neurobiol. Dis. 2005, 20, 666-672.

[39] Lim, M. K., Kawamura, T., Ohsawa, Y., Ohtsubo, M. et al., Parkin interacts with LIM Kinase 1 and reduces its cofilinphosphorylation activity via ubiquitination. Exp. Cell Res. $2007,313,2858-2874$

[40] Xu, J., Kao, S. Y., Lee, F. J., Song, W. et al., Dopamine-dependent neurotoxicity of alpha-synuclein: a mechanism for selective neurodegeneration in Parkinson disease. Nat. Med. 2002, 8, 600-606.

[41] Kawamoto, Y., Akiguchi, I., Nakamura, S., Honjyo, Y. et al., 14-3-3 proteins in Lewy bodies in Parkinson disease and diffuse Lewy body disease brains. J. Neuropathol. Exp. Neurol. 2002, 61, 245-253.
[42] Sato, S., Chiba, T., Sakata, E., Kato, K. et al., 14-3-3eta is a novel regulator of parkin ubiquitin ligase. EMBO J.2006, 25, 211-221.

[43] Jin, K., Mao, X. O., Cottrell, B., Schilling, B. et al., Proteomic and immunochemical characterization of a role for stathmin in adult neurogenesis. FASEB J. 2004, 18, 287-299.

[44] Gavet, O., Ozon, S., Manceau, V., Lawler, S. et al., The stathmin phosphoprotein family: intracellular localization and effects on the microtubule network. J. Cell Sci. 1998, 111, 3333-3346.

[45] Strey, C. W., Spellman, D., Stieber, A., Gonatas, J. O. et al., Dysregulation of stathmin, a microtubule-destabilizing protein, and up-regulation of Hsp25, Hsp27, and the antioxidant peroxiredoxin 6 in a mouse model of familial amyotrophic lateral sclerosis. Am. J. Pathol. 2004, 165, 1701-1718.

[46] Jin, L. W., Masliah, E., limoto, D., Deteresa, R. et al., Neurofibrillary tangle-associated alteration of stathmin in Alzheimer's disease. Neurobiol. Aging 1996, 17, 331-341.

[47] Gosavi, N., Lee, H. J., Lee, J. S., Patel, S., Lee, S. J., Golgi fragmentation occurs in the cells with prefibrillar alphasynuclein aggregates and precedes the formation of fibrillar inclusion. J. Biol. Chem. 2002, 277, 48984-48992.

[48] Fujita, Y., Ohama, E., Takatama, M., Al-Sarraj, S., Okamoto, K., Fragmentation of Golgi apparatus of nigral neurons with alpha-synuclein-positive inclusions in patients with Parkinson's disease. Acta Neuropathol. 2006, 112, 261-265.

[49] Goedert, M., Alpha-synuclein and neurodegenerative diseases. Nat. Rev. Neurosci. 2001, 2, 492-501.

[50] Lavedan, C., Leroy, E., Dehejia, A., Buchholtz, S. et al., Identification, localization and characterization of the human gamma-synuclein gene. Hum. Genet. 1998, 103, 106-112.

[51] Galvin, J. E., Uryu, K., Lee, V. M., Trojanowski, J. Q., Axon pathology in Parkinson's disease and Lewy body dementia hippocampus contains alpha-, beta-, and gamma-synuclein. Proc. Natl. Acad. Sci. USA 1999, 96, 13450-13455.

[52] Uversky, V. N., Li, J., Souillac, P., Millett, I. S. et al., Biophysical properties of the synucleins and their propensities to fibrillate: inhibition of alpha-synuclein assembly by betaand gamma-synucleins. J. Biol. Chem. 2002, 277, 1197011978.

[53] Fearnley, J. M., Lees, A. J., Ageing and Parkinson's disease: Substantia nigra regional selectivity. Brain 1991, 114, 22832301. 